4. Discussion of the results. In the case of the two dimensional channel the basic Poiseuille flow causes a discharge obtained from (3) of amount $2 a u_{m} /$ unit width of channel. Thus if we confine ourselves to one pulse the total discharge at time $T$ is

$$
a u_{m}\left\{2+4 A_{0} H(T / \pi) \sum_{m=0}^{\infty} \exp \left[-\left(m+\frac{1}{2}\right)^{2} T\right]\right\} \text {. }
$$

Since the pulses act in a direction opposing the free streaming $A_{0}$ is negative and if $T$ is such that

$$
\frac{1}{2}<-A_{0} \sum_{m=0}^{\infty} \exp \left[-\left(m+\frac{1}{2}\right)^{2} T\right]
$$

then the flow will be reversed. This inequality can clearly be satisfied if $T$ is small. The length of time for which the flow is arrested will depend on the magnitude of the impulse coefficient $A_{0}$. Alternatively, if another impulse follows the first rapidly enough, the flow will remain arrested for a further period.

In the case of the circular pipe the basic flow causes a discharge $\pi a^{2} u_{m}$. So the total flux due to a single pulse and the basic flow is

$$
a^{2} u_{m}\left(\pi+2 \pi A_{0}\left\{2 \sum_{m=1}^{\infty} \exp \left(-\alpha_{m}^{2} T\right) H(T)+\left[\frac{1}{2}-2 \sum_{m=1}^{\infty} \alpha_{m}^{-2} \exp \left(-\alpha_{m}^{2} T\right)\right] \delta(T)\right\}\right) .
$$

It follows, as before that when $T$ is small but not zero the flow will be arrested if

$$
\frac{1}{4}<-A_{0} \sum_{m=1}^{\infty} \exp \left(-\alpha_{m}^{2} T\right) .
$$

Conclusions. The above analysis shows that it is entirely possible for the flow of a viscous fluid, in a pipe or channel, to be arrested when the pipe is subjected to one or more pulses of sufficient strength. The time for which stoppage occurs depends, essentially, on the magnitude of the pulses, the viscosity of the fluid and the radius of the pipe as well as on the frequency of the pulses.

\title{
REFERENCE
}

1. B. van der Pol and H. Bremmer, Operational calculus, Cambridge University Press, 1950.

\section{AN EXTENDED UPPER BOUND THEOREM ON THE ULTIMATE LOADS OF BUCKLED REDUNDANT TRUSSES ${ }^{1}$}

By E. F. MASUR (University of Michigan)

Introduction. This note is intended to supplement an earlier paper ${ }^{2}$ in which the collapse load of a buckled redundant rigid-jointed truss was bracketed between a lower and an upper bound. As was pointed out at that time, the lower bound theorem permits the computation of a sequence of "statically admissible" parameters which approach the

${ }^{1}$ Received August 22, 1955. This paper was written under the sponsorship of the Office of Ordnance Research, Contract No. DA-11-022-ORD-1156 while the writer was at Illinois Institute of Technology.

${ }^{2}$ E. F. Masur, Lower and upper bounds to the ultimate loads of buckled redundant trusses, Quart. Appl. Math. 11, 385-392 (1954). 
exact value of the ultimate load within arbitrarily close limits. The upper bound theorem, on the other hand, restricts itself to the establishment of a finite (usually small) set of load parameters, of which each is known to be too large. The gap between lower and upper bound can therefore not be narrowed down arbitrarily; furthermore, the lowest of all upper bounds is usually not close enough to the exact collapse load to be of technical interest.

It is easy to see why this should be so. In fact, the proof of the upper bound theorem is predicated on the construction of a series of collapse modes which are physically unrealistic since they involve the concept of some bars buckling as fixed-end columns, while the remaining bars are rigid. This set of collapse modes without joint rotations does not include the actual collapse mode; this accounts for the shortcomings of the upper bound principle described above.

An extended upper bound theorem. In what follows, the concept of a collapse mechanism will be extended in such a way as to encompass an infinity of collapse modes. With each mode there is associated a "kinematically admissible" load parameter. Moreover, the actual collapse mode belongs to the set of modes to be defined; hence, the ultimate load parameter is also kinematically admissible. Finally, it will be the object of this note to establish the following.

TheоRem. The ultimate load parameter is the smallest of all kinematically admissible load parameters.

To prove this principle, let a system of bar forces $S_{k}$ be in equilibrium with the external loads identified by the multiplier $\lambda_{0}^{\prime \prime}$, and let a set of geometrically consistent deflection functions $y_{k}(x)$ exist which satisfy the equations of equilibrium

$$
\left(E I y_{k}^{\prime \prime}\right)^{\prime \prime}-S_{k} y_{k}^{\prime \prime}=0 \text {. }
$$

Let it be assumed further that the change-in-length terms

$$
\delta_{k}=\frac{1}{2} \int_{0}^{L_{k}}\left(y_{k}^{\prime}\right)^{2} d x
$$

are derivable from a geometrically consistent set of joint displacements; this is satisfied if, and only if,

$$
\sum_{k} S_{k}^{(r)} \delta_{k}=0 \quad(r=1,2, \cdots, m) .
$$

Let finally the potential energy associated with the configuration $y_{k}(x)$ be non-positive, that is:

$$
\sum_{i} \sum_{i} a_{i i} \theta_{i} \theta_{i} \leqq 0 .
$$

When these conditions are complied with, the multiplier $\lambda_{0}^{\prime \prime}$ will be designated as being "kinematically admissible." It is readily apparent that the collapse load parameter $\lambda_{0}^{u}$ is itself kinematically admissible since, under collapse conditions, Eqs. (1), (2), and (3) as well as the equality sign in (4) are satisfied.

In a manner similar to a previous analogous development ${ }^{3}$ the satisfaction of the

${ }^{3}$ E. F. Masur, A large deflection theory of redundant structures, Proc. Second U. S. Nat. Congr. Appl. Mech., 427-436 (1955). 
first three equations can be shown to be tantamount to the single set of equations

$$
\sum_{i} \sum_{i} a_{i j, r} \theta_{i} \theta_{i}=0 \quad(r=1,2, \cdots, m) .
$$

Hence the load parameter $\lambda_{0}^{\prime \prime}$ fulfills the condition of kinematic admissibility if there exists a system of internal forces $S_{k}$ (in equilibrium with the external loads associated with $\lambda_{0}^{\prime \prime}$ ) and a set of joint rotations $\theta_{i}$ such that the relations (4) and (5) are satisfied. ${ }^{4}$

If now the truss, with its force system so defined, is deformed into a kinematically admissible configuration satisfying Eqs. (1), (2), and (3), it follows from the force equations of equilibrium at the joints and the compatibility of the bar shortenings $\delta_{k}$ that

$$
\lambda_{0}^{\prime \prime} W=-\sum_{k} S_{k} \delta_{k}
$$

in which $\lambda_{0}^{\prime \prime} W$ represents the work done by the external loads. Similarly, for the same configuration, the actual collapse force system $S_{k}^{u}$ (corresponding to the collapse parameter $\lambda_{0}^{u}$ ) gives rise to the work relationship

$$
\lambda_{0}^{u} W=-\sum_{k} S_{k}^{u} \delta_{k}
$$

Hence, by combining Eqs. (6) and (7),

$$
\sum_{k}\left(S_{k}^{u}-S_{k}\right) \delta_{k}=\left(\lambda_{0}^{\prime \prime}-\lambda_{0}^{u}\right) W \text {. }
$$

It can readily be demonstrated by means of a few partial integrations and in view of the geometric boundary conditions that

$$
U_{B}+\sum_{k} S_{k} \delta_{k}=\frac{1}{2} \sum_{i} \sum_{i} a_{i j} \theta_{i} \theta_{i}
$$

with $U_{B}$ defined as the bending strain energy. On the other hand, it follows from the neutrality of the equilibrium associated with $S_{k}^{u}$ and $\lambda_{0}^{u}$ that

$$
U_{B}+\sum_{k} S_{k}^{u} \delta_{k} \geqq 0 ;
$$

in (10), the equality sign applies non-trivially only to the actual collapse mode. Therefore, upon substitution of (9) and (10) in Eq. (8),

$$
\left(\lambda_{0}^{\prime \prime}-\lambda_{0}^{u}\right) W \geqq-\frac{1}{2} \sum_{i} \sum_{i} a_{i j} \theta_{i} \theta_{i} \geqq 0,
$$

where the second inequality is the result of (4).

No generality is sacrificed by postulating a positive parameter $\lambda_{0}^{\prime \prime}$; this implies also $W>0$ by Eqs. (6) and (9), the inequality (4), and the positive definiteness of $U_{B}$. It follows therefore from (11) that

$$
\lambda_{0}^{\prime \prime} \geqq \lambda_{0}^{u}
$$

which completes the proof of the theorem.

In closing it may be pointed out that once an upper bound $\lambda_{0}^{\prime \prime}$ to the collapse load is obtained, a lower bound may be found, for instance, by reducing all bar forces in the same proportion until, corresponding to, say, $\lambda_{0}^{\prime}$, neutral equilibrium is reached. It is known then that the actual collapse multiplier $\lambda_{0}^{u}$ is bounded between $\lambda_{0}^{\prime}$ and $\lambda_{0}^{\prime \prime}$.

${ }^{4}$ It is of course to be remembered that the stiffness matrix $\left[a_{i j}\right]$ is a function of the force system $S_{k}$. 